\section{Tropical Journal of Pathology and Microbiology}

\title{
Evaluation of markers of thrombosis and fibrinolysis in malaria patients in tertiary care hospital in Central India
}

DOI: https://doi.org/10.17511/jopm.2020.i08.07

1* Swati Chouhan, Consultant, Department of Pathology, Apex Hospital, Varanasi, Uttar Pradesh, India.

2 Siddharth Gautam Singh, Senior Resident, Department of E.N.T, ESIC Hospital, Varanasi, Uttar Pradesh, India.

Background: Cases of malaria are known to be associated with variable degrees of coagulopathy as evident from abnormalities of screening coagulation assays in a large number of patients during the illness. However, attempts to use laboratory parameters as indicators of the severity of the underlying coagulopathy and for monitoring disease progression have met with only partial success. Methods: This prospective study was conducted in the department of Pathology in Ruxmaniben Deepchand Gardi Medical College, Ujjain. Blood samples of all the patients diagnosed positive with malaria were collected in vials containing ethylene tetra-acetic acid tri potassium salt for hematological parameters and in trisodium citrate tubes for coagulation assays. Result: Out of the total positive cases, PT was prolonged in $65 \%$ of total cases, Percentage positivity for prolonged PT for falciparum and vivax is $60 \%$ and $68.5 \%$ respectively. APTT has raised $47 \%$ of malaria positive cases with percentage positivity for falciparum and vivax $48 \%$ and $45.7 \%$ respectively. $55 \%$ of total cases showed raised levels of fibrin degradation products, which included $48 \%$ of falciparum cases and $60 \%$ of vivax cases. Out of 120 positive cases of malaria, D- Dimer was raised $41.7 \%$ cases. Conclusion: As blood is the chief component affected by malaria, not only proper examination of peripheral smears is required for detection and species identification, but a careful evaluation of other laboratory parameters should be done irrespective of the malarial species for the early diagnosis of compensatory and non-compensatory consumptive coagulopathy.

Keywords: Malaria, Fibrinolysis, Platelet, Fibrin degradation product

Corresponding Author

Swati Chouhan, Consultant, Department of Pathology, Apex Hospital, Varanasi, Uttar Pradesh, India.

Email: dr.sgs007@gmail.com
How to Cite this Article

To Browse

Chouhan S, Singh SG. Evaluation of markers of thrombosis and fibrinolysis in malaria patients in tertiary care hospital in Central India. Trop J Pathol Microbiol. 2020;6(8):498-503. Available From

https://pathology.medresearch.in/index.php/jopm/ar ticle/view/490

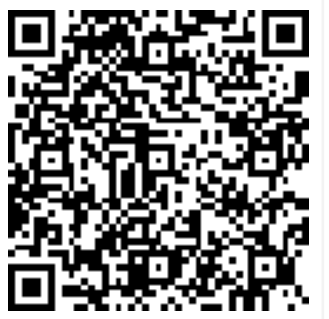

Manuscript Received 2020-10-20

Conflict of Interest No
Review Round 1 2020-10-30

Funding
Review Round 2 2020-11-15

Ethical Approval Yes
Review Round 3

Plagiarism X-checker $7 \%$
Accepted 2020-12-23

Note

(C) 2020 by Swati Chouhan, Siddharth Gautam Singh and Published by Siddharth Health Research and Social Welfare Society. This is an Open Access article licensed under a Creative Commons Attribution 4.0 International License https://creativecommons.org/licenses/by/4.0/ unported [CC BY 4.0]. 


\section{Introduction}

William Osler has said that "Humanity" has but 3 great enemies; fever, famine, and war; of these by far the greatest, by far the most terrible is fever".

Malaria is the most important protozoal parasitic disease of humans. The word malaria derives its origin from the Italian "mal-aria" meaning bad air [1]. WHO estimated worldwide prevalence of malaria is about $300-500$ million per annum. It is present in more than 100 countries around the world. Most of these are developing countries or underdeveloped countries. [2]

Cases of malaria are known to be associated with variable degrees of coagulopathy as evident from abnormalities of screening coagulation assays in a large number of patients during the illness. However, attempts to use laboratory parameters as indicators of the severity of the underlying coagulopathy and for monitoring disease progression have met with only partial success [3]. Therefore, the authors wanted to revisit this subject in a prospective study involving a large number of malaria patients admitted at our tertiary care hospital in Ujjain and see if the platelet count is a good indicator of the underlying vasculopathy and of the hemorrhagic tendency and that normal screening coagulation assays are associated with the absence of hemorrhagic complications.

This study aimed to see if the elevation in D-Dimer levels and fibrin degradation products act as a marker of thrombosis and fibrinolysis respectively. Malaria affects almost all the organs of the body but the chief component affected is blood. Therefore, hematological changes play a major role in malaria pathology. The present study is an effort to correlate the laboratory changes in blood, and if they are the indicator of its clinical outcome.

\section{Materials and Methods}

Place of the study: Department of Pathology in Ruxmaniben Deepchand Gardi Medical College, Ujjain.

Duration and type of the study: The present prospective study was conducted over one and a half years i.e. January 2015 to July 2016.

Ethical considerations and permission: Ethical considerations and permission were met through the Institutional ethical committee.

\section{Inclusion criteria:}

01. All proven patients with malaria admitted in our tertiary care hospital were included.

02. Both male and female patients of any age were included.

03. Cases with mixed malarial infection were also included in the study.

\section{Exclusion criteria:}

01. Cases of co-infection with malaria and dengue will be excluded from the study.

02. Pregnant women will be excluded.

03. Patients with acute hepatitis, leptospirosis, salmonella, and those with other bacterial and viral infections; immuno-compromised individuals, e.g. AIDS and patients having other causes of coagulopathy will be excluded.

Sampling Method: A total of 120 patients were included in the study. Blood samples of all the patients diagnosed positive with malaria were collected in vials containing ethylene tetra-acetic acid tri potassium salt (K3EDTA) for hematological parameters and in trisodium citrate tubes (3.8\%) for coagulation assays. Each blood sample underwent Peripheral blood smear examination, Complete blood counts, Prothrombin time (PT), International normalized ratio(INR), Activated partial thromboplastin time(APTT), Fibrin degradation product (FDP), and D- Dimer. A prestructured proforma was used to collect baseline data.

\section{Result}

During the study period, 120 patients were diagnosed as malaria positive in the hospital, of which $70(58 \%)$ patients were of Plasmodium vivax (P.v) and $50(42 \%)$ patients were of Plasmodium falciparum (P.f). Out of total cases, $72(60 \%)$ patients were males whereas $48(40 \%)$ patients were females i.e, more number of males were affected.

P.v was seen more in males whereas, P.f was seen more in females. Of all the cases $71.6 \%$ cases were observed in the age group of $0-40$ years and $28.3 \%$ cases were observed in the later ages the frequency decreases with increasing age. In the age group of 0 to 20 years equal prevalence of vivax and falciparum was found, however with increasing age the prevalence of falciparum malaria decreased (Table 1). 
Table-1: Age-wise distribution of P. vivax and P. falciparum cases.

\begin{tabular}{|l|l|l|}
\hline \multicolumn{1}{|c|}{ Age } & \multicolumn{1}{c|}{ P. falciparum } & \multicolumn{1}{c|}{ P. vivax } \\
\hline 0 to 10 years & 10 & 10 \\
\hline 11 to 20 years & 12 & 12 \\
\hline 21 to 30 years & 10 & 18 \\
\hline 31 to 40 years & 6 & 8 \\
\hline 41 to 50 years & 6 & 6 \\
\hline 51 to 60 years & 2 & 8 \\
\hline 61 to 70 years & 2 & 8 \\
\hline 71 to 80 years & 2 & 0 \\
\hline
\end{tabular}

$83 \%$ of the total study cases had thrombocytopenia with platelet counts $<1,50,000 /$ cumm, $97 \%$ of $\mathrm{P}$. vivax and $64 \%$ patients of $P$. falciparum showed thrombocytopenia.

38 of total malaria positive patients, platelet counts ranged from 25,000 to $<50,000 / \mu$ l. The $P$ value is 0.000683 which is significant (Figure 1 ).

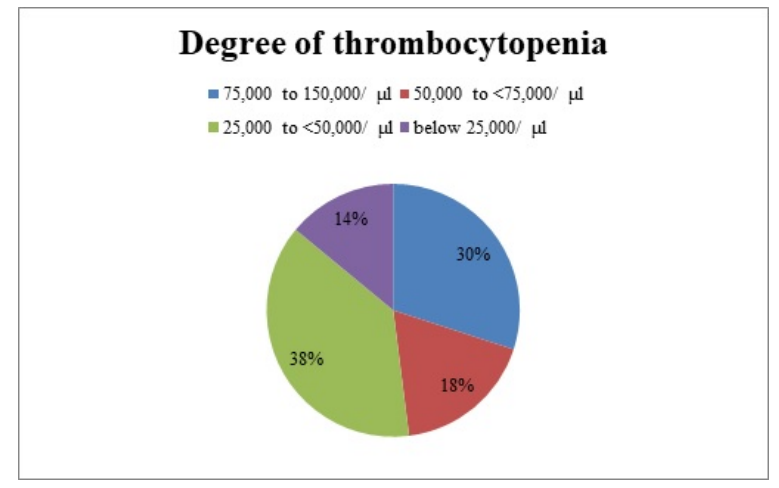

Fig-1: Degree of thrombocytopenia.

Out of the total positive cases, PT was prolonged in $65 \%$ of total cases, Percentage positivity for prolonged PT for P.f and P.V is $60 \%$ and $68.5 \%$ respectively.

APTT was raised in $47 \%$ of malaria positive cases ( $P$ value $>0.05$, not significant) with percentage positivity for P.f and P.v $48 \%$ and $45.7 \%$ respectively.

24 (18 P.v and 6 P.f) of the total malaria cases had isolated prolongation of PT, however, only 2 cases of P.V showed isolated prolongation of APTT. This shows that the extrinsic pathway of coagulation is affected more in malaria as compared to the intrinsic pathway.

The common pathway is affected giving rise to both prolongations in PT and APTT in a significant number of 54 cases (Figure 2).

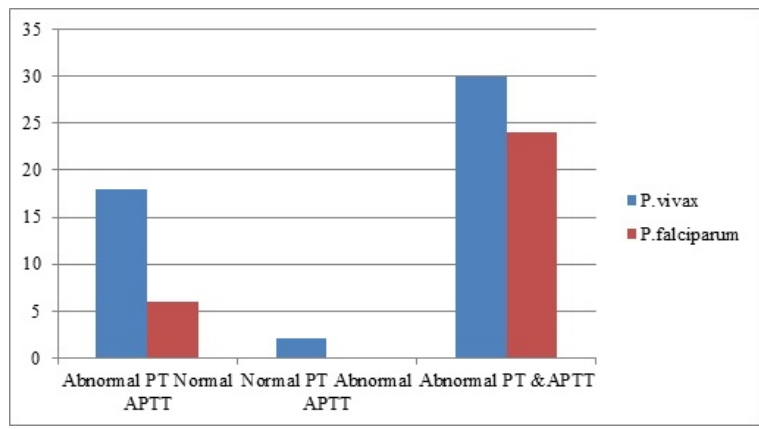

Fig-2: Coagulation parameters in malaria cases.

$55 \%$ of total cases showed raised levels of fibrin degradation products, which included $48 \%$ of falciparum cases and $60 \%$ of vivax cases. Table 2 shows the relationship between different coagulation parameters in cases of malaria. Out of 120 positive cases of malaria, D- Dimer was raised in $50(41.7 \%)$ cases which consisted of $45.8 \%$ of the vivax and $35.7 \%$ of falciparum cases that were tested. The mean hemoglobin of study cases is 9.74 $\mathrm{g} / \mathrm{dl}$, which shows that malaria causes a mild degree of anemia, however, mean hemoglobin of $8.4 \mathrm{~g} / \mathrm{dl}$ was observed in falciparum malaria cases.

(The $\mathrm{t}$ - value is $3.63596, \mathrm{P}$-value is 0.000295 , the result is significant). The average $\mathrm{MCV}$ and $\mathrm{MCHC}$ remain at the lower limit of normal irrespective of the species of malaria. The average platelet count in malaria shows thrombocytopenia and was $88.9 x$ $10^{\wedge} 3 / \mu \mathrm{l}$, but the average platelet count in patients with P.vivax was lower $\left(64.9 \times 10^{\wedge} 3 / \mu \mathrm{l}\right)$ and in the patients with $\mathrm{P}$. falciparum, it was $122.3 \times 10^{\wedge} 3 / \mu \mathrm{l}$. (The $t$ value is $-2.31398, \mathrm{P}$-value is 0.012116 . The result is significant at $p<0.05$ )

Table-2: Relationship of various coagulation parameters.

Relationship of various coagulation parameters

\begin{tabular}{|l|l|}
\hline Thrombocytopenia, PT and APTT Prolonged FDP Positive & 50 \\
\hline Thrombocytopenia, PT and APTT Prolonged FDP Negative & 4 \\
\hline Thrombocytopenia, PT and APTT Normal FDP Positive & 4 \\
\hline Thrombocytopenia, PT and APTT Normal FDP Negative & 18 \\
\hline Thrombocytopenia, PT Prolonged APTT Normal FDP Negative & 12 \\
\hline
\end{tabular}

Table-3: Mean of various haematological parameters.

\begin{tabular}{|l|l|l|l|l|}
\hline Parameters & \multicolumn{1}{|c|}{$\begin{array}{c}\text { HB } \\
(\mathbf{g} / \mathbf{d L})\end{array}$} & $\begin{array}{c}\text { MCV } \\
(\mathbf{f l})\end{array}$ & \multicolumn{1}{|c|}{$\begin{array}{c}\text { MCHC } \\
(\mathbf{g} / \mathrm{dL})\end{array}$} & $\begin{array}{l}\text { Platelet Count (10^3/ } \\
\boldsymbol{\mu l})\end{array}$ \\
\hline Mean (Total) & 9.94 & 80.57 & 33.18 & 88.9 \\
\hline Mean P.v & 11.0 & 81.6 & 33.2 & 64.9 \\
\hline Mean P.f & 8.4 & 79.0 & 33.0 & 122.3 \\
\hline
\end{tabular}




\section{Discussion}

In the present study, positive cases were observed in all the age groups although a maximum number of malaria cases were observed in the age group of 10 to 30 years and the least cases in the age group of 71 to 80 . The frequency of malaria declined as the age increased. Similar findings were observed in a study carried out by Mohan Kashikunti et al [4] who reported a maximum number of malarial cases in the age group of 20 to 40 years.

The above results suggest that malaria affects the young and the population which is more exposed to the outdoor environment. In the present study, $16.6 \%$ of cases were in the age group of $0-10$ years. These children play outdoors without wearing full clothes and in poor hygienic conditions and thus are vulnerable to mosquito bites.

In the present study, $52 \%$ of cases were of P. vivax and $48 \%$ of P. falciparum, our results are comparable to the study carried out by Kini Reshma $G$ et al [5], which showed $60 \%$ cases of P. vivax. It corroborates with the fact that P. Vivax is the most common species of malaria in India followed by $P$. falciparum. Studies conducted by Himanshu Shekhar et al [6] and Mohan Kashikunty et al [4] show $54 \%$ and $76 \%$ of falciparum cases which may be due to difference in the geographical distribution of malarial species and show a rise in the incidence of P. falciparum over last few decades with a higher incidence of P.f as compared to P.v.

Anemia was reported in $62 \%$ of cases in the study carried by Himanshu Shekhar et al [6], whereas severe anemia was reported in $16 \%$. Similarly, Sharma et al [7] reported anemia in $86.7 \%$ cases with severe anemia in $10 \%$ cases and Mohapatra et al [8] reported anemia in 59\% of cases respectively. Our results are comparable to the studies carried out by them as anemia was found in $55 \%$ of the cases studied with severe anemia i.e hemoglobin < $6 \mathrm{~g} / \mathrm{dl}$ was seen in $13 \%$ of total cases. The mean hemoglobin value in patients with P. falciparum was $8.4 \mathrm{~g} / \mathrm{dl}$ lower than the mean hemoglobin value for $P$. vivax which was $11 \mathrm{~g} / \mathrm{dL}$ in the present study. ( $P$ value $<0.05$ ).

$83 \%$ of cases in the present study suffered from thrombocytopenia. Studies conducted by Sharma et al [7] who showed thrombocytopenia in $90 \%$ of their cases, Himanshu Shekhar Das et al [6] reported thrombocytopenia in $68 \%$ of cases, and Kini Reshma G et al [5] in $96 \%$ of their cases.
These studies included cases of both vivax and falciparum species of malaria. In the present study, $64 \%$ of the total falciparum cases and $97 \%$ of the total vivax cases who presented with thrombocytopenia ( $P$-value is 0.000683 ) results are statistically significant.

Our results are comparable to the studies reported by Srivastava et al [9] in which thrombocytopenia was more commonly seen in vivax cases. However, our results were not comparable to the study by Himanshu Shekar et al [6] where the incidence of thrombocytopenia was more in falciparum cases and may be due to the high endemicity of $P$. falciparum in the study population.

Various screening tests were used as indicators of coagulation in malarial patients which included PT, APTT, FDP, and D- Dimer in the present study. Prothrombin time is the measure of the integrity of extrinsic and the final common pathway of coagulation. Whereas activated partial thromboplastin time is the indictor for intrinsic and the common pathway of coagulation.

In the present study, Platelets were raised in 70 cases $(65 \%)$, and APTT was raised in 56 cases $(47 \%)$. Our findings are comparable to the studies conducted by Rajniti Prasad et al [10], Dutta et al [11].

Products of degradation of fibrin meshwork by the action of plasmin lead to the formation of crosslinked fibrin degradation products. D-Dimer is the smallest plasmin resistant unit of cross-linked fibrin degradation products. It comprises two $D$ fragments which are linked together.

Estimation of raised FDP and D- Dimer are the markers of thrombotic events such as DIC. The presence of D- Dimer represents the activation of coagulation cascade followed by activation of the fibrinolytic system resulting in the fatal complication of DIC in malaria.

A study conducted by Dutta et al [11] showed FDP positivity in $85 \%$ of malarial cases following the present study where FDP was positive in $55 \%$ of cases. Studies were done by Sahoo A.K and Das A.K et al [12] and Sharma et al [7] also showed raised FDP levels, but only in $6.59 \%$ and $16.7 \%$ cases respectively.

Quantitative estimation of D- Dimer levels were found above the normal levels ( $>200 \mathrm{ng} / \mathrm{dl}$ ) in $41.7 \%$ in the present study. 
94\% cases with positive FDP and all the cases with raised D-Dimer levels (P-value $<0.05$ )in the present study had platelet counts $<1,00 \times 10^{\wedge} 3$ / cumm suggesting that thrombocytopenia plays a critical role in the pathogenesis of thrombosis and fibrinolysis in malarial patients irrespective of the species involved causing DIC. These figures are comparable with a study conducted by Anirban Das Gupta et al [13].

It was observed that thrombocytopenia was seen in all the cases with deranged coagulation parameters. However, our findings were not in correlation with Ladhani et al [14]. They reported that thrombocytopenia was not the sole cause of DIC but other autoimmune mechanisms and increased peripheral destruction of platelet due to oxidative stress are also possible. However, they suggested that detailed platelet kinetic studies will be required to establish such observation.

$5 \%$ of malaria cases in the present study presented with bleeding manifestations in the course of the disease and all had severe thrombocytopenia (platelet count $<20 \times 10^{\wedge} 3 /$ cumm) and elevated PT, APTT, and FDP. These observations were also reported in the studies carried out by Valluri Satya Prasad et al [15], Sahoo A.K, and Das A.K et al [12] with $10 \%$ and $8.8 \%$ cases with bleeding manifestations respectively.

\section{Conclusion}

There are many national programs like NVBDCP (National Vector Borne Disease Control Programme) implemented in India for the control of parasitic diseases like malaria. But, the inability to completely control and eliminate malaria, and it still exists as a burden, especially to economically reproductive ages.

\section{What does the study add to the existing knowledge?}

As blood is the chief component affected by malaria, not only proper examination of peripheral smears is required for detection and species identification, but a careful evaluation of other laboratory parameters including platelet count, PT, APTT, FDP, and if required D- Dimer level estimation should be done irrespective of the malarial species for the early diagnosis of compensatory and non-compensatory consumptive coagulopathy and the life-threatening bleeding manifestations.

\section{Author's contribution}

Dr. Swati Chouhan: Concept, study design

Dr. Siddharth Gautam Singh: Manuscript preparation

\section{Reference}

01. RW Colman, Jack Hirsh, Victor J Marder, and Edwin W Salzman. Haemostasis and Thrombosis- Basic Principles and Clinical Practice. 3rd Ed, JB Lippincott Company. 1994;1197-1206.

[Crossref]

02. Kenneth J, Arrow, Claire B. Panosian and Hellen Gelband, Chloroquine and quinine-institute of medicine of the national academics.

[Crossref]

03. CRC. Handbook Series in Clinical Laboratory, Science, Section 1- Haematology. CRC Press, Inc Boca, Raton Florida. 1980;III.

[Crossref]

04. Mohan K, Shruthi A. Clinical, hematological and coagulation proflile in malaria. Sch J Appl Med Sci. 2014;2(2B)584-588.

[Crossref]

05. Kini Reshma G, Lobo Veronica, Lyngdoh Raphael $\mathrm{H}$, MsVedasree R. Clinical Correlation of Coagulopathy in Vivax Malaria, Published by Pacific Group of e-Journals (PaGe). AABS3. (1) 2016. [Crossref]

06. Das HS. Study of clinical and laboratory profile in malaria. Indian J App Res. 2015;5(12)218222.

[Crossref]

07. Sharma SK, Das RK, Das BK, Das PK. Haematological and coagulation profile in acute falciparum malaria. ] Assoc Physicians India. $1992 ; 40 ; 581-583$.

[Crossref]

08. Mohapatra S, Samantaray JC, Arulselvi S, Ghosh A. Disseminated intravascular coagulation following malaria due to Plasmodium vivax- $A$ thromboelastography-based study. Malar J. $2013 ; 12 ; 336$.

doi: $10.1186 / 1475-2875-12-336$ [Crossref] 
09. Srivastava S, Ahmad S, Shirazi N, Kumar Verma $S$, Puri P. Retrospective analysis of vivax malaria patients presenting to tertiary referral centre of Uttarakhand. Acta Trop. 2011;117(2)82-85.

doi: 10.1016/j.actatropica.2010.10.001 [Crossref]

10. Prasad R, Das BK, Pengoria R, Mishra OP, Shukla J, Singh TB. Coagulation Status and Platelet Functions in Children with Severe Falciparum Malaria and their Correlation of Outcome. J Trop Pediatr. 2009;55(1)374-378.

doi: $10.1093 /$ tropej/fmp028 [Crossref]

11. Datta S, Roul LD, Das S, Panda JK, Pattanayak NC. A study of the coagulation profile in falciparum malaria. Orissa physicians journal OPJ. $2011 ; 7 ; 13-17$.

[Crossref]

12. Sahoo AK, Das KK. Coagulation profile in severe falciparum malaria and its clinical correlation. Int J Gen Med Pharmacy. 2013;2(1)9-14.

[Crossref]
13. Dasgupta A, Rai S, Das Gupta A. Persistently Elevated Laboratory Markers of Thrombosis and Fibrinolysis After Clinical Recovery in Malaria Points to Residual and Smouldering Cellular Damage. Indian J Hematol Blood Transfus. $2012 ; 28(1) 29-36$.

doi: $\quad 10.1007 / \mathrm{s} 12288-011-0106-y \quad$ [Crossref]

14. Ladhani S, Lowe B, Cole AO, Kowuondo K, Newton CR. Changes in white blood cells and platelets in children with falciparum malariarelationship to disease outcome. Brit J Haematol. 2002;119(3)839-847.

doi: $10.1046 / j .1365-2141.2002 .03904 . x$ [Crossref]

15. Misra DP, Das S, Pattnaik M, Singh SC, Jena RK. Relationship of hepatic and renal dysfunction with haemorrheological parameters in Plasmodium falciparum malaria. J Assoc Physicians India. 2011;59;552-556.

[Crossref] 